\title{
Multimessenger astrophysics (with a take on dark matter)
}

\section{Nicolao Fornengo*}

University of Torino, Department of Physics and INFN - Sezione di Torino

E-mail: nicolao.fornengodunito.it

Thanks to the recent observational and theoretical advancements, multimessenger astrophysics is becoming a necessary tool to study dark matter. Although we currently do not have a unique and obvious target for the dark matter particle, we can rely on a broad range of ideas, tools and methods that make the investigation of dark matter a multi-frequency, multi-messenger and multitechniques integrated endeavour.

Neutrino Oscillation Workshop

4 - 11 September, 2016

Otranto (Lecce, Italy)

${ }^{*}$ Speaker. 


\section{Astrophysical messengers of dark matter}

Depending on its mass and interactions, dark matter (DM) $[1,2,3,4,5]$ can produce a large variety of astrophysical messengers, than can reach us and therefore be used to probe its particle physics nature. Let us recall that DM needs to be dynamically relatively cold, in order to form the observed large scale structure of the Universe: this implies that DM signals produced by DM annihilation cannot have energies larger than the DM mass (or half its mass, in case of DM decay). This fact sets the target of investigation for the different DM candidates. Weakly interacting massive particles (WIMPs), with masses in the $\mathrm{GeV}$ to few $\mathrm{TeV}$ range, can produce energetic neutrinos, photons in the gamma-ray band and energetic electrons and positrons. They can also produce an exotic component of antiprotons and heavier antinuclei, most notably antideuterium [6] and antihelium [7]. Moreover, whenever WIMPs produce electrons and positrons, these latter can suffer secondary electromagnetic effects, and produce an additional component of gamma-rays, as a consequence of the up-scatter of the cosmic microwave background (CMB) radiation and other radiation fields through inverse-Compton scattering. Furthermore, ambient (galactic or extragalactic) magnetic fields can induce electrons and positrons to emit in the radio band through the synchrotron process. Inverse Compton can also be responsible of an X-ray continuum emission. Therefore, WIMPs can be searched for with a full multi-wavelength and multi-messenger approach. In addition to these opportunities, that collectively represent the indirect search technique and test the self-annihilation or decay DM processes, the local component of galactic WIMPs can also directly interact with a passive low-background detector located in underground laboratories: in this case (direct search), the signal is the deposited energy in the detector and the tested interaction is the one between DM and ordinary matter (typically nuclei, but also electrons, especially for light DM) [8].

Lowering the mass of the DM particle makes some channels unavailable, while others remain open. MeV DM can therefore contribute (in most models though its decay) a low-energy electron and positron flux, with its inverse Compton and synchrotron emissions producing electromagnetic radiation again from the $\mathrm{X}$ ray down to the radio band. Lighter particles, like e.g. keV sterile neutrinos, can only produce photons (or standard neutrinos): the $\mathrm{X}$ ray and infrared bands are typically the most investigated. Even lighter particles, like axions, can only produce low-frequency photons, in the microwave or radio bands. While the CMB is a dominant radiation field at microwave frequecies, it is nevertheless well understood: a DM emission in this frequency range can potentially emerge as a distortion of the perfect CMB black-body spectrum. Astrophysical radio emission away from the CMB peak is more erratic, but DM decay contributes in a way that is morphologically and/or spectrally different from radio astrophysical sources.

CMB can be used also to study WIMPs or other heavier particles: if DM (again) annihilates or decays into electrons and positrons, these particles can distort the CMB spectrum: this can happen, for instance, in a galaxy cluster, where the large amount of DM can produce a distribution of nonthermal electrons, such that Compton scattering distorts the CMB passing through the cluster (it's a Sunyaev-Zeldovich effect due to relativistic electrons) [9]. The same can occur if DM produces electrons in the early Universe, contributing an ionization component that can distort the CMB and be felt in the CMB temperature anisotropies at large cosmological scales [10].

In most models, also neutrinos are a produced signal, but they become increasingly more difficult to detect when their energy decreases, and in some energy range they also have overwhelming 
backgrounds, like in the case of the 1-10 MeV range, where solar neutrinos are a dominant irreducible flux. Neutrinos as messengers of the presence of dark matter are typically (although not exclusively) investigated when they are at or above the atmospheric neutrinos energy range, and therefore when they can be produced by WIMPs or even heavier DM particles.

In the following, due to space constraints, we will briefly report on the most novel technique, namely cross-correlations between gamma rays and a gravitational tracer of dark matter.

\subsection{Cross-correlations}

Being the sum of many independent sources (either DM halos or astrophysical sources), the DGRB is to first approximation isotropic. But at a deeper level anisotropies are clearly present. This means that, even though sources are too dim to be individually resolved, they can affect the statistics of photons across the sky. Recently, different techniques have been proposed to investigate the unresolved components of the gamma-rays sky. DM emission is actually among the dimmest contributors: these new methods, based on statistical analyses, can therefore be a turning point in the identification of a signal.

The first method is based on a 1-point statistics estimator, the so-called "photon pixel count", based on the fact that different sources, with varying levels of emission and distribution in the sky, contribute a different number of photons to the various pixels of a gamma-rays map [11]. This technique has been used on the Fermi/LAT data, to derive the number count distribution of faint gamma-ray point-sources: this is a first step that allows to better understand the astrophysical populations of gamma-rays emitters, and will now be extended to DM investigation. The same technique, plus another advanced statistical method [12], has also been used to test whether the galactic center gamma-rays "excess" is originated by a a true diffuse emission (which would point toward a DM interpretation) or instead if it is compatible with a distribution of point sources [13].

The second technique looks at the Fermi/LAT gamma-ray sky map by constructing its 2-point correlator $[14,15]$. The gamma-ray angular power spectrum has been measured, including its energy dependence. It appears compatible with a population of point sources just below the detection threshold. The energy dependence also points to a hint for the presence of two different source populations, one dominating below $10 \mathrm{GeV}$ and one above. Being compatible with point sources, no evidence of a DM signal is present in the angular power spectrum: this allowed to set bounds on WIMP DM [15]. These bounds less constraining than the bounds coming directly from the integrated DGRB, but are obtained from an observable that feels how DM is distributed in the Universe.

A potentially more powerful technique is the cross-correlation between an electromagnetic DM signal (e.g., gamma rays) and a gravitational tracer of DM in the Universe [16, 17, 18]. This technique has been proposed recently and it exploits two distinct features of particle DM, one referring to its particle physics nature and one that is a direct evidence of the presence of DM. The best gravitational tracer is a lensing observable, since it measures directly, in an unbiased way, the presence of DM and where it is. Cosmic shear is the most direct option, since it directly traces the whole DM distribution. CMB lensing traces DM imprints on the CMB anisotropies, and is more sensitive to high redshift. Alternatively, gravitational tracers can be galaxy or cluster catalogs. In this case, the gravitational tracer is biased, since it traces light and not directly DM, but on the other 
hand the statistics of detected objects can be large, and it will be even more so in the future, since galaxy surveys are one of the major current and planned endeavours in cosmology.

A positive cross-correlation between Fermi/LAT gamma-ray maps and a host of galaxy catalogs (2MASS, SDSS, NVSS) has been detected [19]. This can be very well explained by DM, with a mass (around $100 \mathrm{GeV}$ mass) and interactions (close to thermal) in the interesting range form WIMPs [20] Although at the moment it's not statistically possible to disentangle a pure DM contribution from a blazars component, nevertheless, the results show that this technique is potentially able to identify a DM signal even though the total gamma-ray DM emission is largely subdominant in the total DGRB emission. This shows the potentiality of the cross-correlation technique [20]. More recent investigations can be found in Ref. [21].

Recently, a positive signal has been measured also by correlating cluster catalogs with the Fermi/LAT gamma rays maps [22]. Concerning the cross-correlation with weak lensing observables, at the moment the cosmic shear catalogs are still covering small portions of the sky and no signal has been detected [23]. In the next years, especially with the Dark Energy Survey (DES) data and, in a farther future, with Euclid, this technique will definitely be able to detect a positive correlation, which will then be posed under scrutiny to identify whether it refers to astrophysical sources (therefore gaining insight in the type and distribution of gamma-rays emitters) or if instead a DM component is present. It has been shown that under favourable conditions, DES could be able not only to identify a signal, but also to infer the particle physics parameters of DM (its mass and interactions) [16]. Euclid will then have the possibility to either pinpoint the DM particle properties or to significantly disprove the WIMP hypothesis [16]. What is clear is that we have to exploit all opportunities: one option does not fit all possibilities, and to understand what DM is requires to plan with the idea to explore all alternatives. A positive cross-correlation has also been detected between Gamma-rays and CMB lensing: this correlation arises from higher redshift, as compared to the one measured with galaxy catalogs. Since DM emits its electromagnetic signals mostly at low redshift, galaxy and cluster catalogs and cosmic shear are the places where to put under scrutiny the DM hypothesis; CMB lensing instead can provide a leverage to constrain the astrophysical emission, and therefore reduce the uncertainty on this component in the large-scale-structure and weak-lensing cross-correlation.

\section{Conclusions}

Multimessenger astrophysics offers a wide range of opportunities to study DM in most its full mass range: from axions and axion-like particles, to sterile neutrinos and majorons, to WIMPs and non-thermal alternatives in the $\mathrm{GeV}$ to $\mathrm{TeV}$ mass range, all the way to super-heavy DM. Astrophysical fore/back-grounds are very complex and typically dominant over the sought-after DM signals: this implies that a clever identification of potential targets, signatures and signal features is necessary. DM searches will in fact progress together with a better understanding and modelling of the astrophysical environment, and together with the identification and development of new methods and techniques (like, e.g., the statistical approach of cross-correlations, recently proposed). Up to know, some intriguing hints have been identified, but to establish that any of these clues are indeed originated by DM will require further investigation, deeper understanding of the astrophysical settings and, possibly, independent confirmation in a different exploration channel. The field 
is progressing rapidly and will profit from a large wealth of data expected in the next 5-15 years, that will make the investigation of DM as an elementary particle even more exciting and hopefully conclusive.

\section{Acknowledgments}

Work supported by the research grant Theoretical Astroparticle Physics number 2012CPPYP7 under the program PRIN 2012 funded by the Ministero dell' Istruzione, Università e della Ricerca (MIUR), by the research grants TAsP (Theoretical Astroparticle Physics) and Fermi funded by the Istituto Nazionale di Fisica Nucleare (INFN).

\section{References}

[1] Jungman, Kamionkowski, Griest, Phys. Rep. 267 (1996) 267; Bertone, Hooper, Silk, Phys. Rep. 405 (2005) 279; Schmaltz, Tucker-Smith, Annual Review of Nuclear and Particle Science 55 (2005) 229; Hooper, Profumo, Phys. Rep. 453 (2007) 29; Feng, ARA\&A 48 (2010) 495.

[2] Ade et al (Planck Collaboration), A\&A 594 (2016) A13.

[3] Navarro, Frenk, White, Ap. J. 490 (1997) 493.

[4] Klypin et al, Ap. J. 522 (1999) 82; Moore, Nature 370 (1994) 629 (1994); Flores and Primack, Ap. J. 427 (1994) L1; Boylan-Kolchin et al, MNRAS 415 (2011) L40; Gentile et al, MNRAS 351 (2004) 903; Donato and Salucci, MNRAS 353 (2004) L17.

[5] Mac Low, Ferrara, Ap. J. 513 (1999) 142; Governato et al, Nature 463 (2010) 203; J. Silk, Nusser, Ap. J. 725 (2010) 556 (2010); Wang et al, MNRAS 424 (2012) 2715; Governato et al, MNRAS 422 (2012) 1231; Brooks et al, Ap. J. 765 (2013) 22; Zolotov et al, Ap. J. 761 (2012) 71; Amorisco et al Ap. J. 782 (2014) L39.

[6] Donato, Fornengo, Salati, Phys. Rev. D 62 (2000) 04300.

[7] Carlson et al, Phys. Rev. D 89 (2014) 076005; Cirelli, Fornengo, Taoso, Vittino, JHEP 1408 (2014) 009.

[8] For a recent review on the status of direct searches (and indirect searches, as well) see the Proceedings of TAUP 2015, Journal of Physics: Conference Series 718 (2016) (Editors: Fornengo, Regis, Zechlin).

[9] Colafrancesco, A\&A, 422 (2012) L23.

[10] Zhang et al, Phys. Rev. D 76 (2007) 061301; Galli et al, Phys. Rev. D 80 (2009) 023505; Slatyer et al, Phys. Rev. D 80 (2009) 043526; Kanzakiet et al, Prog. Theor. Phys. 123 (2010) 853; Hisanoet et al, Phys. Rev. D 83 (2011) 123511; Hutsi et al, A\&A 535 (2011) A26; Galli et al, Phys. Rev. D 84 (2011) 027302; Finkbeiner et al, Phys. Rev. D 85 (2012) 043522; Slatyer et al, Phys. Rev. D 87 (2013) 123513; Galli et al, Phys. Rev. D 88 (2012) 063502; Lopez-Honorez et al, JCAP 1307 (2013) 046; Madhavacheril et al, Phys. Rev. D 89 (2014) 103508; Slatyer, Phys. Rev. D 93 (2016) 023527.

[11] Malyshev, Hogg, Astrophys. J. 738 (2011) 181; Zechlin et al, Ap. J. Suppl. 225 (2015) 039; Zechlin, et al, Ap. J. Lett. 826 (2016) 831; Lisanti et al, Ap. J. 832 (2016) 117.

[12] Bartels, Krishnamurthy, Weniger, Phys. Rev. Lett. 116 (2016) 05102. 
[13] Lee et al, JCAP 1505 (2015) 056; Lee et al, Phys. Rev. Lett. 116 (2016) 051103.

[14] Ando, Komatsu, Phys. Rev. D 73 (2006) 02352; Ando, Komatsu, Narumoto, Totani, Phys. Rev. D 75(2007) 063519; Cuoco et al, JCAP 0704 (2007) 013; Cuoco et al Phys. Rev. D 77 (2008 )123518; Siegal-Gaskins, JCAP 0810 (2008) 040; Siegal-Gaskins, Pavlidou, Phys. Rev. Lett. 102 (2009) 241301; Ando, Phys. Rev. D 80 (2009) 023520; Fornasa et al, Phys. Rev. D D80 (2009) 023518; Taoso et al, Phys. Rev. D 79 (2009) 043521; Ibarra et al, Phys. Rev. D 81 (2010) 023529; Hensley et al, Ap. J. 723 (2010) 277; Zavala et al, MNRAS 405 (2010) 593; Cuoco et al, MNRAS 414(2011) 2040; Campbell, Dutta, Phys. Rev. D 84 (2011) 075004; Fornasa et al, MNRAS 429 (2012) 1526; Ando, Komatsu, Phys. Rev. D 87 (2013) 123539; Campbell, Beacom, arXiv:1312.3945 Fornengo, Regis, Front. Physics 2 (2014) 6; Gomez-Vargas et al, NIM A742(2014) 149; Ando et al, MNRAS 376 (2007) 1635.

[15] Ackerman et al (Fermi Collaboration), Phys. Rev. D 85 (2012) 083007; Fornasa et al, Phys. Rev. D 94, 123005 (2016).

[16] Camera, Fornasa, Fornengo, Regis, Ap. J. Lett. 771 (2013); Camera, Fornasa, Fornengo, Regis, JCAP 06 (2015) 029.

[17] Cuoco et al, Phys. Rev. D 77 (2008 )123518; Ando, Benoit-Levy, Komatsu, Phys. Rev. D 90 (2014) 023514; Fornengo, Regis, Front. Physics 2 (2014) 6; Ando, JCAP 1410 (2014) 061; Xia, Cuoco, Branchini, Fornasa, Viel, MNRAS 416 (2011) 2247; Xia, Cuoco, Branchini, Viel, Ap. J. Suppl. 217 (2015) 15; Regis, Xia, Cuoco, Branchini, Fornengo, Viel, Phys. Rev. Lett. 114 (2015) 24130; Cuoco, Xia, Regis, Branchini, NF, Viel, Ap. J. Suppl. 221 (2015) 29.

[18] Fornengo, Perotto, Regis, Camera, Ap. J. Lett. 802 (2015) 1 L1.

[19] Xia, Cuoco, Branchini, Viel, Ap. J. Suppl. 217 (2015) 15.

[20] Regis, Xia, Cuoco, Branchini, Fornengo, Viel, Phys. Rev. Lett. 114 (2015) 241301; Cuoco, Xia, Regis, Branchini, Fornengo, Viel, Ap. J. Suppl. 221 (2015).

[21] Shirasaki, Horiuchi, Yoshida, Phys. Rev. D 92 (2015) 123540.

[22] Cuoco, Regis, Camera, Branchini, Fornengo, Viel, Xia, arXiv:1612.05788.

[23] Shirasaki, Horiuchi, Yoshida, Phys. Rev. D 90 (2014) 063502; Shirasaki, Macias, Horiuchi, Shirai, Yoshida, Phys. Rev. D 94, 063522 (2016); Troester, arXiv:1611.03554. 